\title{
Long-term outcomes of microvascular decompression and Gamma Knife surgery for trigeminal neuralgia: a retrospective comparison study
}

\author{
Andras A. Kemeny $^{1}$ (D) \\ Received: 28 August 2017 / Accepted: 4 September 2017 / Published online: 18 September 2017 \\ (C) Springer-Verlag GmbH Austria 2017
}

Inoue and his colleagues retrospectively reviewed their experience with MVD and GK treatment for trigeminal neuralgia. They conclude that the long-term (which they defined as beyond 1 year) outcome is better after microsurgery than after radiosurgery. While this opinion is shared by many, and some available data in the literature do support it, one would be right to question whether this article actually proves it. In my opinion, their study highlights inherent weaknesses of such a study and acknowledging the weaknesses of the study does not negate them.

The main difficulty comparing an open surgical technique with a closed procedure relates to recruitment. Their study collected data from two consecutive time periods. While one may think that their patient selection remained unchanged, this is far from self-explanatory. Their own views taking on a patient referred to them may have changed, and the referring doctors may have altered their attitudes. Importantly, patient acceptance may have evolved. Even in randomised controlled trials, supposedly free from admission bias, one finds that patients come with pre-conceived ideas about what they should have and those with strong views do not sign up for a study. In their scenario some patients may not have even come to them in the second period who would have wanted to be referred when radiosurgery was a likely choice. Proof for this, in the material presented by Inoue et al., is the age difference between the two cohorts. Gamma Knife treatment will have been easier to accept in the elderly than open surgery.

In their material, Inoue et al. ended postoperative evaluation when the patient had any additional surgical intervention.

Andras A. Kemeny

aakemeny@gmail.com

1 Thornbury Radiosurgery Center, Sheffield, UK
This is potentially a misleading endpoint. Using the neurological status immediately before the additional treatment is unreliable when it is the decision to offer further treatment that is up to bias. This remains a true weakness of the paper. Given that a second microvascular decompression would be a significantly higher risk undertaking, there would be a higher threshold for "additional surgical treatment" after a first microvascular decompression, unless a prospective decision has been made to make any "second procedure" a crossover, i.e. offering radiosurgery. I note from correspondence with the authors that those who now decline open surgery are sent back to pain clinics (i.e. back to medical treatment rather than GKS).

When assessing these patients, one should acknowledge that they just want to be rid of their pain, preferably without side effects. The often used method, the BNI score, excludes a very common outcome: those with no pain on a minimal dose of well-tolerated medication. A significant cohort of patients choose to carry on with a very low dose to avoid the occasional sensations they associate with a potential future relapse. It is often difficult to convince them even to have a trial period without the protection of their favourite medication. Most of these individuals titrate their own medication to achieve a balance. It is a bias against radiosurgery to describe these patients as treatment failures or those in whom treatment effect was not recognised: this is often a very satisfied group.

Trigeminal neuralgia will remain a condition where individualised management is the gold standard rather than one or another technique. Very careful history, examination and sympathetic interview about expectations are as important as radiological studies. Predetermined choice of intervention, governed by the customs (or availability) in a particular department, may not be the best way forward. 